\title{
Ultériorité dans le passé et conditionnels en basque navarro-labourdin
}

\author{
Georges Rebuschi*
}

\begin{abstract}
Résumé L'ultériorité en basque continental peut être marquée soit par un suffixe aspectuel prospectif (qui s'oppose au perfectif et à l'imperfectif) sur les participes verbaux, soit par le suffixe - $k e$ sur le verbe (lexical ou auxiliaire) fléchi - mais - $k e$ peut aussi avoir, selon le contexte, des valeurs modales diverses, qui vont de l'équipossibilité à la prédiction engageant le locuteur, en passant par la probabilité. Du côté des marques de temps et d'accord, on a aussi trois dimensions : le présent $(d-)$ s'oppose à la fois au passé $(z-)$ et à l'éventuel $(l-)$, que l'on trouve dans les conditionnels du présent-futur. Il n'y a donc pas d'identification possible entre l'expression de l'ultériorité dans le passé (participe prospectif + aux. au passé) et celle du conditionnel présent-futur - mais c'est cette même combinaison qui traduit le plus souvent notre conditionnel passé ou contre-factuel, et qui peut enfin, depuis plus d'un siècle, correspondre au would dit fréquentatif de l'anglais, ce qui peut s'expliquer par une réanalyse modale du suffixe de prospectif.
\end{abstract}

Mots-clés ultériorité; conditionnel passé; modalités.

[Version du 04/04/2012]

\section{English title and abstract Ulteriority in the Past and Conditionals in Navarro- Laboudin Basque}

Abstract Northern Basque has two strategies to denote ulteriority: (i) by a prospective aspectual ending on participles, and (ii) by inserting the morpheme -ke, which can also express various epistemic shades, from equipossibility to probability to certainty in the finite verb form. From the viewpoint of morphological "tense", three 3rd p. prefixes contrast: $d$ - (present), $z$ - (past) and $l$-, which occurs in present / future conditionals. Ulteriority in the past is thus expressed by associating the prospective aspect and an auxiliary in the past, and therefore can never be used as a non-past conditional. However, past, counterfactual conditionals are generally expressed by the same [prospective+past] combination, which also happens to correspond to a marked habitual meaning, roughly corresponding to English frequentative would, probably due to a reanalysis of the prospective suffix as a modal one.

Key-words ulteriority; past conditional; modality.

$$
* * *
$$

\section{INTRODUCTION ${ }^{* *}$}

En basque, ce n'est pas le conditionnel présent-futur, mais le conditionnel passé qui est réalisé par une construction qui exprime l'ultériorité dans le passé. De plus, cette forme, de manière paradoxale, permet aussi d'exprimer l'habitude.

En 2. est introduite la conjugaison simple, ainsi qu'un affixe modal que l'on trouve dans l'expression de l'ultériorité et de certains conditionnels. En 3., les conjugaisons composées et les conditionnels non-passés correspondants sont décrits, puis, en 4., les constructions périphrastiques qui dénotent l'ultériorité, au passé comme au présent. Enfin, en 5., les différentes expressions du conditionnel passé seront étudiées, puis on montrera ce qui permet à la combinaison prospectif+passé de prendre aussi une valeur fréquentative.

\footnotetext{
* Sorbone-Nouvelle \& LACITO. Courriel : georges.rebuschi1 @ free.fr

** Je remercie Marie Pourquié, Beñat Oyharçabal et les relecteurs anonymes de FDL pour leurs remarques sur diverses versions antérieures de ce travail.
} 


\section{LA CONJUGAISON SIMPLE (OU SYNTHETIQUE)}

Le basque possède une conjugaison synthétique pour quelques verbes lexicaux dits «forts», parmi lesquels izan 'être' et ukan 'avoir' (ou izan aussi en labourdin), qui servent aussi d'auxiliaires dans la conjugaison composée, et enfin des constructions proprement périphrastiques pour l'expression modalisée de l'ultériorité.

\subsection{La conjugaison synthétique: passé, présent}

Deux formes proprement temporelles s'opposent: le présent, marqué par le préfixe de $3^{\mathrm{e}}$ p. $d$-, et le passé, marqué par le préfixe de $3^{\mathrm{e}}$ p. $z$ - et le suffixe $-(e) n$; les préfixes varient aussi aux autres personnes, cf., pour le verbe joan 'aller':

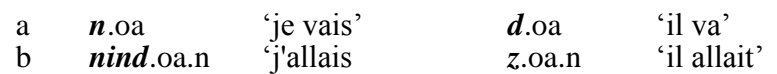

Ainsi conjugués, ces verbes sont interprétés comme progressifs. Noter la différence dans les préfixes de $1^{\mathrm{e}}$ et $2^{\mathrm{e}}$ p., en fonction du temps et du contexte morphologique: si le verbe est transitif, au présent, les préfixes codent le COD, (2a), mais, au passé, si le COD est de $3^{\mathrm{e}}$ p. et le sujet de $1^{\mathrm{e}}$ ou $2^{\mathrm{e}}$ p., le premier disparaît et c'est le second qui est maintenant indiqué par un préfixe, $(2 \mathrm{c})$ :

$$
\begin{array}{ll}
\text { a } & \text { na.rama. } \varnothing \text { 'il m'emporte', b ninde.rama.n 'il } m \text { 'emportait' } \\
\text { c } & \text { ne-rama.n 'je l'emportais' }
\end{array}
$$

\subsection{Le morphème -ke}

Les FVF (formes verbales fléchies) peuvent prendre un suffixe, -ke, usuellement décrit comme potentiel (Lafitte 1962), mais il a en fait presque toutes les valeurs épistémiques, de l'équi-possibilité au certain en passant par la probabilité, ou encore une valeur «orientée énonciateur» de supputation, de conjecture, de prédiction, voire plus, comme le montre l'ex. suivant, avec 'avoir' comme verbe lexical et où la double valeur de futur et d'engagement de l'énonciateur est indéniable: Saririk ez $_{2}$ dukezue 3 zuen Aita $_{4}$ zeruetan denaren baithan ${ }_{5}$ [Dv 1865], 'Vous $\mathrm{n}_{2}$ 'aurez $z_{3}$ point de récompense ${ }_{1}$ auprès de votre Père ${ }_{4}$ qui est aux cieux $_{5}$ ' (Mt 6,1) (id. dans [LL 1946], [Ezk 1974]). Ce ke- peut aussi s'affixer à un verbe au passé, avec toutes les nuances décrites à l'instant; par ex., en (3), la source de la modalisation conjecturale n'est évidemment pas le narrateur omniscient, mais le référent du sujet (marqué par -te-dans la FVF zituzketen) :

(3) Kalerna ${ }_{1}$ hurbildua zuten 2, eta $_{3}$ ihurzuria $_{4}$ gainean $_{5}$ zituzketen $_{6}$ laster $_{7}$.

'L'orage ${ }_{1}$ s'était rapproché $_{2}$, et $t_{3}$ ils allaient avoir / auraient 6 bientôt ${ }_{7}$ des coups de tonnerre ${ }_{4}$ au-dessus 5 [de leur tête].'

[Bar 1929]

\subsection{L'éventuel (I) : présent-futur synthétique}

Un troisième préfixe, $l$-, «éventuel » (terme de Lafon 1970-73), n'apparaît que dans des FVF préfixées de $b a$ - (suppositif, dans la protase) ou suffixée de -ke, formant ainsi le conditionnel proprement dit $^{1}$, i.e. la forme de l'apodose :

(4) Ba.l.aki $i_{1}$ zer mugaz 2 ethor daiteken ohoina $_{4}$, atzarririk l.ego.ke $_{6}$.

' $S$ 'il savait ${ }_{1}$ à quelle heure allait arriver $_{3}$ le voleur 4 , il resterait éveillé $_{5}$.' 1974]. [Hrt 1855] : Mt 24,43 - Idem dans [Dv 1859], [LL 1946] ou [Ezk

En conjugaison synthétique, ces formes en $l$ - ont deux caractéristiques importantes : elles ne font jamais référence à un monde passé, mais, morphologiquement, elles présentent les mêmes préfixes qu'au passé, pour les pers. 1 et 2, ce qui marque dans les deux cas la rupture avec le référentiel de l'énonciation:

$$
\begin{array}{llll}
\text { a ba.ninde.rama } & \text { 's'il } m \text { 'emportait', } & \text { nind.rama.ke 'il m'emporterait' } \\
\text { b ba.ne.rama } & \text { 'si je l'emportais' } & \text { ne.rama.ke 'je l'emporterais' }
\end{array}
$$

\footnotetext{
${ }^{1} \mathrm{Si}$ le basque emploie bien le conditionnel pour exprimer un souhait ou un conseil sans heurter l'interlocuteur, la valeur de médiativité lui est par contre totalement inconnue.
} 


\section{LES CONJUGAISONS ANALYTIQUES (OU COMPOSEES)}

\subsection{Les formes marquées aspectuellement, présent et passé}

Morphologiquement, trois «aspects » s'opposent : le perfectif, l'imperfectif, et le prospectif; quand l'auxiliaire (qui suit le participe dans les assertions positives) est au présent, on obtient ainsi le futur ordinaire, (6c) ci-dessous :

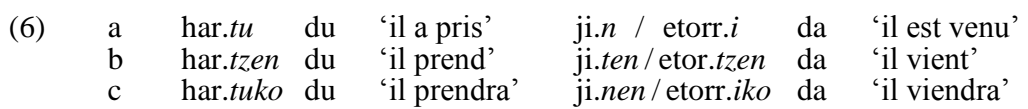

La parenté sémantique entre les formes synthétiques en -ke et celles, analytiques, avec le prospectif, est illustrée par ces deux traductions tirées de l'Imitation de Thomas a Kempis $(2,8,3)$ : 'Jésus sera/restera avec vous', Jesus egonen da zurekin [Dv 1896], Jesus zurekin badagoke ${ }^{2}$ [LL 1929].

Quand l'aux. est au passé et l'aspect est le perfectif, on obtient le temps de la narration, cf. (6a). Avec l'imperfectif, on a l'équivalent de l'imparfait français. Par contre, la combinaison prospectif + passé offre plusieurs valeurs, dont (7d), option marquée, non notée dans les grammaires, par rapport à l'imperfectif :

$\begin{array}{llr} & \text { Har.tuko [prendre-PROSP] } & \text { z.u.en AUX-PASSE :ABS3SG-ERG3SG } \\ \text { a } & \text { 'il le prendrait / allait le prendre' } & \text { (ultériorité dans le passé) } \\ \text { b } & \text { '(je lui ai demandé) s'il le prendrait'3 } & \text { (question indirecte) } \\ \text { c } & \text { 'il l'aurait pris' } & \text { (conditionnel passé contre-factuel) } \\ \text { d } & \text { 'il le prenait' } & \text { (habitude, action typique) }\end{array}$

La première interprétation, (7a), va de soi. Considérons ainsi l'extrait (8), à propos de la chasse aux sorcières au Pays basque au $17^{\mathrm{e}}$ siècle :

(8) (Hola zenaz geroz, gizonak aski zuen...bere eginbidea betetzea.). De

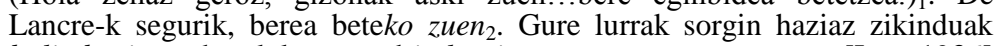
balin baziren $_{3}$, harek laster garbituko zituen 4 ... [Larz 1936] '(Puisqu'il en était ainsi, il suffisait à l'homme de remplir son devoir. $)_{1}$ De Lancre, à coup sûr, allait remplir 2 le sien. Si/comme nos terres étaient polluées $_{3}$ par la semence des sorcières, lui les nettoierait ${ }_{4}$ rapidement.' $^{\prime}$

$\mathrm{Au}$ présent, on aurait les futurs composés beteko du 'il remplira' et zikinduak (balin) ba-dira, ... garbituko ditu 'si/comme elles sont polluées, il les nettoiera.

(7b) illustre le discours indirect, avec la principale au passé (qui impose la concordance des temps). Le suffixe -en du passé est ici «absorbé » par le suffixe -en des questions indirectes et des relatives, quel qu'en soit le temps (il l'est aussi par la marque des complétives des verbes de dire, -(e)la), cf. l'ex. de la note 9).

Nous reviendrons évidemment sur les valeurs (c) et (d) de (7) en 5.

\subsection{L'éventuel (II) et les conditionnels (II, III) : verbe lexical et auxiliaire en l-}

Au présent-futur, et avec un verbe marqué aspectuellement, la seule combinaison usitée est la suivante, où l'ultériorité est marquée deux fois dans l'apodose :

$$
\begin{array}{ll}
\text { Ikusten [IMPER.] ba.l.u, hartuko [PROS.] l.u.ke } \\
\text { 'S'il le voyait, }
\end{array}
$$

A côté des verbes marqués aspectuellement, il existe une autre conjugaison analytique, qui associe radicaux verbaux et auxiliaires défectifs (ils n'ont pas de forme non-fléchie). J'appelle ces formes «virtuelles » en raison de leur contenu, et aussi parce qu'elles ne peuvent pas être employées sans affixation supplémentaire. Ainsi, *har d.eza/z.eza/l.eza ne sont énonçables qu'une fois pourvus soit d'un suffixe de subordination, donnant des subjonctifs, soit du suffixe -ke, ce qui donne de vrais potentiels : har-Ø d.eza.ke / z.ezake.(e)n 'il peut / pouvait le prendre'. Avec $l$-, on obtient un binôme conditionnel aoristique : ikus- Ø ba-l.eza 's'il le voyait'/har- Ø l.eza.ke 'il le prendrait' - qui ne s'oppose guère aux formes de (9). Comme ces conditionnels présent-futur n'ont d'autre rapport avec l'ultériorité que la présence de -ke, et, avec le passé, que la parenté morphologique de la préfixation, je n'y reviendrai pas.

\footnotetext{
${ }^{2}$ Le préfixe $b a$ - ici n'a qu'une valeur assertive, et se distingue du $b a$-suppositif par le fait qu'il est tonique, le second étant par contre inaccentué.

${ }^{3}$ Un second suffixe, $-e(t) z$, serait très naturel dans ce contexte.
} 


\section{LES PERIPHRASES EXPRIMANT L'ULTERIORITE}

Il y a trois principales constructions périphrastiques qui expriment l'ultériorité, tant au présent qu'au passé. Elles s'opposent à la conjugaison composée par une plus grande liberté dans l'ordre des mots, et par le fait qu'être' et 'avoir' s'y comportent comme des verbes lexicaux. La première est reliée à un emploi circonstanciel de but d'une forme verbale nominalisée, non-fléchie, en - $t(z) e-k o$, comme dans : Harriak hartu zituzten $_{2}$, Jesusen harrika $_{4} \cdot$ tzeko $_{5}{ }^{4}$ [Ezk 1974], 'Ils ramassèrent $_{2}$ des pierres $_{1}$ pour $_{5}$ lapider $_{4}$ Jésus $_{3}$ ' (Jn 8,31). La seconde option consiste en l'emploi du lexème behar 'devoir/nécessité', qui régit le p. perfectif, et qui, avec les intransitifs «inaccusatifs », s'utilise soit avec 'être' soit avec 'avoir'. La troisième possibilité est offerte par le verbe 'aller' en conjugaison simple, régissant une forme déverbale circonstancielle à l'allatif, -tze-ra(t); elle apporte une nuance d'imminence (l'interprétation peut être littérale dans d'autres contextes). La FVF peut bien sûr être au passé. Les traductions qui suivent illustrent l'équivalence entre ces trois tours - et le futur dans le passé en (11a) :

(10) Mt 2,4 : 'Il (leur) demanda où devait naître le Messie.'

a $\quad \ldots$ non sortzekoa zen.
b $\quad \ldots$ non sorthu behar zuen. [Dv 1859, LL 1946, Edy 2008]
c $\ldots$ non sorthu behar zen...
[Hrt 1865]

(11) Lk 19,11: (ils pensaient) qu'il [le royaume de Dieu] allait apparaître aussitôt.

b ... agertzera zihoala [Hr 1855, Dv 1859]

c .... ethortzekoa zela

[LL 1946]

\section{LE CONDITIONNEL (IV) : CONDITIONNELS PASSES ET FREQUENTATIF PASSE}

\subsection{Le prospectif dans le passé et le conditionnel passé analytique}

On a vu que les FVF en $l$ - font référence à une situation éventuelle. Elles sont donc normalement incompatibles avec le passé, et c'est bien le cas quand il s'agit des conditionnels proprement dits, dans les apodoses - car dans les protases, la marque de la condition associe toujours un participe perfectif (réanalysé comme passé) et un aux. en ( $b a-) l$-, avec une éventuelle duplication de l'auxiliaire renforçant l'antériorité de la condition : ikusi (ukan) balu 's'il l'avait (eu) vu'.

Dans les apodoses donc, on l'a vu avec (7c), ce sont les marques de l'ultériorité dans le passé qui traduisent notre conditionnel passé. Cette double valeur de tours comme hartuko zuen, 'il allait le prendre / il l'aurait pris' s'explique bien : il s'agit d'exprimer la subséquence (ultériorité temporelle sans plus, ou suite ou conséquence logique ou naturelle ${ }^{5}$ ) par rapport à un repère révolu (marqué par l'aux. au passé) - même si l'événement repère n'a pas eu lieu (ou a eu lieu si la protase est négative) : la non-réalisation dudit événement repère est alors marquée dans la protase, où elle s'oppose à une FVF au passé, comme dans l'ex. (8) supra où, si l'on avait (zikinduak) balire 'si elles avaient été polluées' au lieu de baziren, la principale garbituko zituen se traduirait par 'il les aurait nettoyées'. 6

Le navarro-labourdin a deux variantes pour exprimer le conditionnel passé, outre $\mathrm{A}$, hartuko zuen, à savoir: $\mathrm{B}$, hartuØ zuke(e)n (où la subséquence est marquée par -ke sur l'auxiliaire), et $\mathrm{C}$, hartuko zuke(e)n, où elle est doublement marquée. La fréquence relative des ces formes est indiquée par les traductions de : 'Il aurait bien mangé...' datées de 1895 et publiées dans [Aur \& V 2004] : A, type de loin le plus fréquent : 95 traductions sur 113 ; B, 9/113; C., 7/113.

Par ailleurs, outre le type A, seul le type B peut avoir d'autres valeurs. Ainsi, en (12), la conjecture ou probabilité rétrospective est évidente :

(12) Berrondon holako zerbait $_{2}$ pasatu zateken $_{3}$ duela ehun bat urte ${ }_{4}$, ezen bertsu hau gelditu $\mathrm{da}_{5}$ :... [Laf 1934] 'Quelque chose de semblable ${ }_{2}$ avait dû se passer à Berrondo ${ }_{1}$ il y a une centaine d'années ${ }_{4}$, car cette strophe est restée ${ }_{5} \ldots$...'

\footnotetext{
${ }^{4}$ Ce suffixe est distinct de celui du prospectif, qui ne peut s'accorder. De plus, ce $-k o$ circonstancialisant (puis adjectivisé par accord en nombre) n'alterne jamais avec -en, cf. etorr.i.ko mais ji.n.en en (6c). Enfin, le part. prosp. ne peut pas régir le génitif pour le COD, ce qui contraste avec la finale génitive -en de Jesus-en dans l'exemple proposé.

${ }^{5}$ Dans un ex. comme (i), seule la conséquence survit, l'ultériorité temporelle cédant le pas à la simultanéité (voir également (4) pour le présent-futur) :

(i) $\mathrm{Peio}_{1}$ egon $_{2}\left(\right.$ izan) balitz $_{3}$, Imanol ere ${ }_{4}$ egonen zen 5 .

'Si Peio ${ }_{1}$ était ${ }_{3}$ resté ${ }_{2}$, Imanol aussi ${ }_{4}$ serait resté $_{5}$.'

${ }^{6}$ Se rappeler l'insistance de Tesnière (1958/69) sur l'idée que la notion de conditionnel est fondamentalement phrastique dans une langue comme le français, où la flexion est segmentable : ce point de vue s'applique aussi très clairement au basque pour le passé.
} 


\subsection{Le conditionnel passé synthétique}

On a vu en 3.1 que le suffixe -ke, qui marque l'ultériorité dans la conjugaison synthétique, correspond au morphème prospectif dans la conjugaison composée, soit, pour 'être' et 'avoir' respectivement: izanen da $\approx$ datekelditake ${ }^{\diamond 7}$ 'il sera'; ukanen $d u \approx d u k e$ 'il aura'. Si ces auxiliaires sont au passé (soit zuen et zen), la version synthétique peut donc évidemment exprimer le conditionnel passé: izanen zen $\approx$ zateken/ziteken, ukanen zuen $\approx$ zuken. Exemples :

(13) Bigarren hegal hoi izan balitz $_{2}$ lehenbizikoa bezen luzea ${ }_{3}$, luzeago ${ }_{4}$ ziteken $_{5}{ }^{\circ}$ gure belearen ixtoria $a_{6} \ldots$ [Bar 1926-29] 'Si cette cette deuxième aile ${ }_{1}$ [du corbeau d'un conte] avait été ${ }_{5}$ aussi longue que la première ${ }_{3}$, l'histoire de notre corbeau ${ }_{6}$ aurait été $_{5}$ plus longue 4. .'

(14) Jn 15,22: (Si je n'étais pas venu...,) ils auraient été sans tache. (Baldin ethorri izan ez banintz...,) hobenik etzuketen $_{2}$

lit. '(...) ils n'auraient pas eu de faute $_{1}$ ' [Dv 1959, LL 1946, Ezk 1974]

On retrouve ces formes dans les tours périphrastiques, par exemple avec behar ci-dessous : en (a), avec 'être', et en (b) avec 'avoir', conjugués synthétiquement, (c) fournissant deux paraphrases analytiques, où le suffixe prospectif est d'abord directement joint à behar, puis attaché à l'auxiliaire, lui-même participial :

(15) Heb 9,26: il aurait d̂̂ souffrir plusieurs fois...

a Behar $_{1}$ zitekeen ${ }_{2}$ Kristok $_{3} \operatorname{askotan}_{4}$ jasan zezan $_{5} \ldots$

lit. 'Il aurait été ${ }_{2}$ nécessaire ${ }_{1}$ que le Christ $_{3}$ souffrît $_{5}$ souvent $_{4}$.'

b sofritu izan behar zuken ...

lit. 'il aurait dû avoir souffert..'

c pairatu beharko zuen / behar izanen zuen...

[Edy 1992 / 2008]

Dans d'autres contextes, ces formes correspondent à la prédiction, cf. (4) supra, ou encore à la probabilité, comme ci-dessous :

(16) a Arno hura xaharra $_{2}$ ziteken $_{3}{ }_{3}$, ezen egarria $_{5}$ phiztu zion ${ }_{6} \ldots$

b 'Ce vin devait être $_{3}$ vieux $_{2}$, car $_{4}$ il lui aiguisa la soif $_{5}$, Goiz batez le $_{1}$, muthiko bat 2 , hamabortz urtheren ingurua ${ }_{3}$ zukena $_{4}$

Azkaingo bidean haya-haya $\operatorname{zohan}_{5} \ldots$

[El J-B 1888]

'Un matin ${ }_{1}$, un garçon ${ }_{2}$ qui devait avoir ${ }_{4}$ autour de quinze ans ${ }_{3}$ marchait d'un bon pas sur le chemin d'Ascain ${ }_{5}$.'

\subsection{Le conditionnel passé analytique et la valeur fréquentative}

Comme annoncé sous (7d), le type A peut également marquer une habitude - fait qui, à ma connaissance, n'a jamais été noté ${ }^{8}$ :

(21) Hek ikhusiz etxerat bihurtzen ${ }_{1}$, bild $u k o$ ziren 2 herriko haur guziak z $_{3}$ heien inguruetarat 4 . Aita-bitxik , besainka $_{6}$, aurthikiko zuen $_{7}$ ahur bat sos 7 . [Elis 1890] 'En les voyant [le parrain et la marraine, après un baptême] retourner chez $e_{1} x_{1}$, tous les enfants du village se rassemblaient 2 autour d'eux . Le parrain $_{5}$ lançait $t_{7}$ à la volée 6 une poignée de sous 7 .'

(22) Mathieu jauna, sortzetik erakaslea ${ }_{1}$. Bazakien $_{2}, \ldots$ noiz ginen akituak 3 , Pinp !

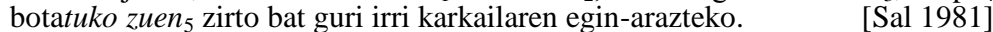

'M. Mathieu [était] un instituteur né ${ }_{1}$. Il savait ${ }_{2}$ quand nous étions fatigués ${ }_{3}$, (et) pouf $!_{4}$, il lançait ${ }_{5}$ une petite blague pour nous faire éclater de rire.'

L'imperfectif, non-marqué, suffirait : ce qu'apporte le prospectif ici, plus qu'une pure ultériorité (par rapport au retour à la maison, ou à la perception de la vue des enfants, respectivement), c'est le caractère naturel, et typique, du lien entre les circonstances et le contenu objectif de la proposition qui le contient : il s'agit donc d'une réinterprétation modale de l'aspect qui permet l'expression d'une prédiction rétroactive dépendant des circonstances, ce qui n'est pas sans rappeler le would dit «fréquentatif» de l'anglais, langue dans laquelle une phrase comme If Peter came, John would stay est ambiguë: (i) '( $\mathrm{Si}$ P. venait,) J. resterait' (conditionnel présent-futur), ou (ii) '... J. restait' (fréquentatif passé typifiant, marquant une particularité de John dans telle ou telle situation).

\footnotetext{
${ }^{7}$ diteke est une neutralisation entre les formes d'izan et d'*edin (l'aux. défectif) au profit du second. Cet emploi des formes d'*edin sera indiqué par le signe «" suivant la FVF. ${ }^{8}$ Le basque n'est pas la seule langue qui associe ainsi l'expression de l'irréel dans le passé et celle de l'habitude, cf. Lazard (1975), qui baptise cette association sémantique "catégorie de l'éventuel » et cite des langues indo-iraniennes, l'arménien, et le géorgien.
} 


\section{CONCLUSIONS}

L'ambiguïté de formes comme ukanen zuen et zuke(e)n ne relève peut-être que de la traduction ('il allait avoir, devait avoir, aurait eu'), car elles marquent chacune la subséquence ou la conjecture par rapport au passé, que ce dernier soit réel, ou irréel (puisque, rappelons-le, c'est la forme d'une subordonnée, explicite ou non, qui précise cette dimension).

Cela dit, il existe trois autres variantes que les types A, B et C, toutes en l-...-ke, et que le navarro-labourdin n'utilise (presque) pas - et il faudrait un travail approfondi pour comprendre pourquoi. Ce sont, pour 'il l'aurait pris', les types $\mathrm{D}$, hartu luke, (23) ci-dessous (avec un préfixe ergatif, cf. (5)), E, hartu luke(e)n, (24), et F, hartuko luke(e)n, (24) :

(23) Bazinakite ${ }_{1}$ zer den hitz hau '...', etzindukete nihoiz $_{4}$ hobengaberik knondenatu $_{6}$. labourdin, [Dv 1859] 'Si vous saviez ${ }_{1}$ ce que signifie[nt] ce $[\mathrm{s}] \operatorname{mot}[\mathrm{s}]_{2}, \ldots$, vous n'auriez $_{3}$ jamais ${ }_{4}$ condamné d'innocents $_{5}$.' (Mt 12,7)

(24) Nurk $_{1}$ sinhetsi $_{2}$ lükien $_{3} \ldots$... Abrahamek erranen ahal ziela ... souletin, [Ar 1862] 'Qui ${ }_{1}$ aurait $_{3} . .$. cru $_{2}$ qu'Abraham pourrait dire..."9 $^{9}$ (Gen 21,7)

(25) Jaunak gu iltea nai izan ba-leu $u_{1}$, ez leukezan gure eskutik $_{3}$ erre-oparia eta eskaintza $_{4}$ ontzat artuko $_{5}$. biscayen, [Ker 1974] 'Si le Seigneur avait voulu nous tuer, il n'aurait, 2 pas accepté ${ }_{5}$ [lit. reçu comme bon] de nos mains l'holocauste et l'offrande 4 .'

(Juges 13,23)

\section{BIBLIOGRAPHIE $^{10}$}

[An 1828] Anonyme, Jesus-Christo [...]ren Testament Berria. ${ }^{\#}$

[Ar 1862] Archu, J.-B., Moisaren lehen libria, Jenesa deithia.*/"

[Arb 1890] Arbelbide, J-P., Erlisionea.*

[Aur \& V 2004] Aurrekoetxea, G. \& Videgain, X. (eds.), Haur prodigoaren parabola.*

[Bar 1926/29] Barbier, J., Piarres, 1926 (I) - 1929 (II).*

[Dv 1859] Duvoisin, J.-P, Bible edo Testament Zahar eta Berria [...].*

[Dv 1896] Duvoisin, J.-P, Jesu-Kristoren imitazionea, Pau.

[Edy 1979] Etchemendy, M., Hasera, Saint-Sébastien, Elkar.

[Edy 1992] Etchemendy, M., Hebrearrei gutuna [...], Saint-Sébastien, Elkar.

[Edy 2008] Etchemendy, M., 2008, Elizen Arteko Biblia (lapurtar-baxenabartarrez), Urt, Biblia Elkartea \& Belokeko Fraidetxea.

[El J-B 1888] Elissamburu, J.-B., Piarres Adame.*

[Elis 1890] Elissamburu, M., Frantziako hirur errepubliken ixtorioa.*

[Ezk 1974] (collectif anon.), 1974, Jesu Kristoren Berri Ona, Bonloc.

[Hrt 1855] Harriet, M., Iesu-Christo[...] ren Testament Berria [...], Bayonne.

[Ker 1976] Euskal-Biblia (bizkaieraz). Bilbao, Bilboko Elizbarrutiko Gotzaintza.

[Laf 1934] Lafitte, P., Euskal literaturaz [recueil d'articles, 1934-1967].*

Lafitte, P., 1964, Grammaire basque (navarro-labourdin ...), Bayonne, Ikas.

Lafon, R., 1970-73, Le suffixe -ke, -te dans la conjugaison basque, BSL 65-1, p. 184-212 \& 67-1, p. 239-265

[Larz 1936] Larzabal, P., ‘Sorginak Lapurdin', reproduit dans Ipuin eta ixtorio.*

[LL 1929] Léon, L., Jesu-Kristoren Imitazionea. */*

[LL 1946] Léon, L., Jesu-Kristo gure Jaunaren Ebanjelio Saindua, Ustaritz.

Lazard, G., 1975, La catégorie de l'éventuel, in Mélanges linguistiques offerts à Emile Benveniste, Paris, Société linguistique de Paris, p. 347-358.

Tesnière, L., [1958] 1969, Eléments de syntaxe structurale, Paris, Klincksieck.

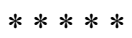

\footnotetext{
9 La version dans [Edy 1979] donne deux combinaisons prospectif + passé pour leurs deux valeurs fondamentales: Nork erranen zuen $_{2}$ Sarak $_{3}$ bularra $_{4}$ emanen zuela semeeri $_{7}$ !, lit. 'Qui ${ }_{1}$ aurait dit ${ }_{2}$ que $\mathrm{Sarah}_{3}$ donnerait $_{5}$ le sein $_{4}$ à [ses] fills 7 !'.

${ }^{10}$ Pour les textes basques téléchargeables, les symboles suivants sont utilisés :

* : textes en rtf, sur le site «Armiarma », http://klasikoak.armiarma.com/alfa.htm

\# : pdf, site «OAI $»:$ http://82.116.160.16:8080/handle/10690/2.

\#\# : pdf, site «Liburuklik» : http://www.liburuklik.euskadi.net/.
} 